\title{
ON THE EXISTENCE OF SOLUTIONS OF SYSTEMS OF FUNCTIONAL DIFFERENTIAL EQUATIONS ${ }^{1}$
}

\author{
JOEL FRANKLIN
}

1. Introduction. We consider the system of equations

$$
\frac{d}{d x} y_{\nu}(x)=f_{\nu}(x, Y(x)) \quad\left(0 \leqq x \leqq x_{1} ; \nu=1, \cdots, n\right),
$$

where the symbol $Y(x)$ stands for the set of functions $\left\{y_{h}\left(u_{k}(x)\right)\right\}$ $(h=1, \cdots, n ; k=1, \cdots, m)$. Under prescribed initial conditions this system is to be solved for the $y$ 's as functions of $x$. The functions $f_{1}, \cdots, f_{n}$ are given, and the functions $u_{1}(x), \cdots, u_{m}(x)$ are also given. For example, in the case of systems of ordinary differential equations we have $m=1$ and $u_{1}(x)=x$, whereas in the case of differential-difference equations, the functions $u_{k}(x)$ are linear functions $x-c_{k}, c_{k} \geqq 0$.

The principal purpose of this paper is to prove an existence theorem analogous to Peano's theorem [1] on ordinary differential equations, in which the only assumption made on the given functions $f_{\nu}$ is that they be continuous functions of their arguments. Our proof will depend on a preliminary theorem which asserts the existence and uniqueness of a solution for the case in which the functions $f_{\nu}$ are restricted to satisfy a Lipschitz condition; this theorem will, of course, be a generalization of the theorem of Picard [2] and Lindelöf [3]. With regard to the preliminary theorem, we note that the Lipschitz condition is by no means the best possible criterion for uniqueness. For sharper uniqueness theorems the reader is referred to [4].

2. Preliminary theorem. First we must define appropriate initial conditions. We assume that the given functions $u_{k}(x)$ are defined and continuous for $0 \leqq x \leqq x_{1}$ and that they satisfy the inequalities

$$
u_{k}(x) \leqq x \quad(k=1, \cdots, m) .
$$

Let the constant $x_{0} \leqq 0$ be defined as $x_{0}=\min \left(x_{01}, \cdots, x_{0 m}\right)$, where

$$
x_{0 k}=\min _{x} u_{k}(x) .
$$

Then let $n$ continuous initial functions $g_{h}(x)$ be given for $x_{0} \leqq x \leqq 0$. In particular, as in the case of differential equations, these functions

Received by the editors June 4, 1953 and, in revised form, October 30, 1953.

1 This work was performed under a contract with the Office of Naval Research. 
are simply initial constants $g_{h}$ if $x_{0}=0$. Let constants $y_{h k}^{0}$ and $y_{h k}^{1}$ be defined as

$$
\stackrel{0}{y_{h k}}=\min g_{h}(x), \quad \stackrel{1}{y_{h k}}=\max g_{h}(x) \quad \text { for } x_{0 k} \leqq x \leqq 0 .
$$

Now let a fixed positive number $p$ be given. Then we suppose that the functions $f_{v}\left(x,\left\{y_{h k}\right\}\right)$ are continuous functions of $x, y_{11}, \cdots, y_{n m}$ for

$$
0 \leqq x \leqq x_{1}, \quad \eta_{h k} \leqq y_{h k} \leqq \zeta_{h k},
$$

where

$$
\eta_{h k}=\min \left[g_{h}(0)-p, \underset{y_{h k}^{0}}{ }\right], \quad \zeta_{h k}=\max \left[g_{h}(0)+p, y_{h k}^{1}\right] .
$$

Now let $M>0$ be chosen so that

$$
\left|f_{p}\left(x,\left\{y_{h k}\right\}\right)\right| \leqq M
$$

for $\nu=1, \cdots, n$ and for the variables $x, y_{11}, \cdots$ ranging in the domain (2). Then we have the following theorem.

TheOREM 1. If $x_{1}$ is $\leqq$ the positive number $p / M$, and if in the domain (2) the functions $f_{\nu}$ satisfy a Lipschitz condition

$$
\sum_{\nu}\left|f_{\nu}\left(x,\left\{y_{h k}\right\}\right)-f_{\nu}\left(x,\left\{z_{h k}\right\}\right)\right| \leqq L \sum_{h, k}\left|y_{h k}-z_{h k}\right|,
$$

where $L$ is a constant $>0$, then the system (1) possesses one and only one set of solutions $y_{1}(x), \cdots, y_{n}(x)$ which are defined and continuous for $x_{0} \leqq x \leqq x_{1}$ and which satisfy the initial conditions

$$
y_{\nu}(x)=g_{\nu}(x)
$$$$
\left(x_{0} \leqq x \leqq 0 ; \nu=1, \cdots, n\right) .
$$

These solutions satisfy the inequalities

$$
\left|y_{\nu}(x)-g_{\nu}(0)\right| \leqq p \quad\left(0 \leqq x \leqq x_{1} ; \nu=1, \cdots, n\right) .
$$

REMARK. Of course, both in this theorem and in Theorem 2, the functions $y_{\nu}(x)$ will be not only continuous for $x_{0} \leqq x \leqq x_{1}$, but also continuously differentiable for $0 \leqq x \leqq x_{1}$, where by the derivative at $x=0$ we mean the right-hand derivative.

Proof. We must solve the system of $n$ equations

$$
y_{\nu}(x)=y_{\nu}(0)+\int_{0}^{x} f_{\nu}\left(t,\left\{y_{h}\left(u_{k}(t)\right)\right\}\right) d t \quad\left(0 \leqq x \leqq x_{1}\right)
$$

under the initial conditions

$$
y_{\nu}(x)=g_{\nu}(x) \quad\left(x_{0} \leqq x \leqq 0\right) .
$$


First we divide the interval $0 \leqq x \leqq x_{1}$ into subintervals by points $0=t_{0}<t_{1}<\cdots<t_{r}=x_{1}$, where

$$
t_{q+1}-t_{q}<\frac{1}{m L} \quad(q=0, \cdots, r-1) .
$$

Then the given system (6) may be solved in $r$ steps:

$$
y_{\nu}(x)=y_{\nu}\left(t_{q}\right)+\int_{t_{q}}^{x} f_{\nu}\left(t,\left\{y_{h}\left(u_{k}(t)\right)\right\}\right) d t \quad\left(t_{q} \leqq x \leqq t_{q+1}\right),
$$

where $q$ is successively equal to $0,1, \cdots, r-1$. Let us suppose that these systems have been solved for $q=0,1, \cdots, s-1$, where $0 \leqq s \leqq r-1$; if $s=0$ and $q=-1$, we mean the trivial system (9) with $t_{-1} \equiv 0=t_{0}$. Let us suppose that a unique set of solutions, $y_{1}(x), \cdots, y_{n}(x)$ has been found for $0 \leqq x \leqq t_{s}$, and that these solutions satisfy the inequalities

$$
\left|y_{\nu}(x)-g_{\nu}(0)\right| \leqq M t_{s} \quad\left(0 \leqq x \leqq t_{s}\right),
$$

where $M$ is the upper bound defined in (3). In particular, we may certainly assume this for $s=0$, in which case we have the degenerate solutions $y_{\nu}=g_{\nu}(0)$. Then we will show that the system

$$
y_{v}(x)=y_{\nu}\left(t_{s}\right)+\int_{t_{s}}^{x} f_{v}\left(t,\left\{y_{h}\left(u_{k}(t)\right)\right\}\right) d t \quad\left(t_{s} \leqq x \leqq t_{s+1}\right)
$$

has one and only one set of solutions $\left\{y_{\nu}(x)\right\}$, and that these solutions satisfy the inequalities

$$
\left|y_{\nu}(x)-g_{\nu}(0)\right| \leqq M t_{s+1} \quad\left(0 \leqq x \leqq t_{s+1}\right) .
$$

If this proof is made for $s=0,1, \cdots, r-1$, then the lemma will be proved.

First let us show that the system (11) has at most one solution. We want first to show that for any solution $\left\{y_{\nu}(x)\right\}$ of (11), the points $y_{h k}=y_{h}\left(u_{k}(x)\right), 0 \leqq x \leqq t_{s+1}$, must lie in the domain (2). Since $M t_{s+1} \leqq M x_{1} \leqq p$, it is sufficient to show that the inequality (12) must hold. Since we are given the inequality (10), it is sufficient to show that

$$
\left|y_{\nu}(x)-y_{\nu}\left(t_{s}\right)\right| \leqq M\left(t_{s+1}-t_{s}\right) \quad\left(t_{s} \leqq x \leqq t_{s+1}\right) .
$$

Let $t^{*}$ be the largest number between $t_{s}$ and $t_{s+1}$ such that

$$
\left|y_{\nu}(x)-y_{\nu}\left(t_{s}\right)\right| \leqq M\left(t_{s+1}-t_{s}\right) \quad \text { for } t_{s} \leqq x \leqq t^{*} .
$$

If $t^{*}<t_{s+1}$, then we must have the inequality sign in (14) for $x=t^{*}$. 
But then

$$
M\left(t_{s+1}-t_{s}\right)=\left|\int_{t_{s}}^{t^{*}} f_{\nu}(\cdots) d t\right| \leqq M\left(t^{*}-t_{s}\right),
$$

so that $t^{*} \geqq t_{s+1}$, which is a contradiction. Hence, we have the inequalities (13) and (12).

Now suppose that $\left\{y_{\nu}(x)\right\}$ and $\left\{z_{\nu}(x)\right\}$ are different solutions of the system (11). Taking account of the fact that $y_{\nu}(t)=z_{\nu}(t)$ for $x_{0} \leqq t \leqq t_{s}$, we find for $t_{s} \leqq x \leqq t_{s+1}$

$$
y_{\nu}(x)-z_{\nu}(x)=\int_{t_{v}}^{x}\left[f_{\nu}\left(t,\left\{y_{h}\left(u_{k}(t)\right)\right\}\right)-f_{\nu}\left(t,\left\{z_{h}\left(u_{k}(t)\right)\right\}\right)\right] d t .
$$

Let

$$
\mu=\max \sum_{\nu}\left|y_{\nu}(x)-z_{\nu}(x)\right| \quad\left(t_{s} \leqq x \leqq t_{s+1}\right) .
$$

Then, using the Lipschitz condition (4), we find

$$
\mu \leqq\left(t_{s+1}-t_{s}\right) L \max _{t_{s} \leqq t \leqq t_{s+1}} \sum_{k=1}^{m} \sum_{h=1}^{n}\left|y_{h}\left(u_{k}(t)\right)-z_{h}\left(u_{k}(t)\right)\right| .
$$

Since $u_{k}(t) \leqq t$, this gives

$$
\mu \leqq\left(t_{s+1}-t_{s}\right) L m \mu .
$$

But, by (8), we have $m L\left(t_{s+1}-t_{s}\right)<1$. Therefore, $\mu=0$ and $y_{\nu}(x)$ $\equiv z_{\nu}(x)$ for $t_{s} \leqq x \leqq t_{s+1}$, as required.

The final step in the proof of the lemma is the construction of the solution set $\left\{y_{v}(x)\right\}$ to the system (11). This is easily done by the method of successive approximations. Let $n$ infinite sequences of functions $y_{v}^{0}(x), y_{v}^{1}(x), y_{v}^{2}(x), \cdots$ (where the upper numbers are superscripts, not exponents) be formed as follows:

$$
\begin{aligned}
y_{\nu}^{N}(x) & =y_{\nu}(x) & & \left(x_{0} \leqq x \leqq t_{s}\right), \\
y_{\nu}^{0}(x) & =y_{\nu}\left(t_{s}\right) & & \left(t_{s} \leqq x \leqq t_{s+1}\right), \\
y_{\nu}^{N+1}(x) & =y_{\nu}\left(t_{s}\right)+\int_{t_{s}}^{x} f_{\nu}\left(t,\left\{y_{h}^{N}\left(u_{k}(t)\right)\right\}\right) d t & & \left(t_{s} \leqq x \leqq t_{s+1}\right) .
\end{aligned}
$$

By the argument used in the preceding proof of the inequality (13), it is immediately clear that these functions $y_{\nu}^{N}(x)$ are defined and continuous for $x_{0} \leqq x \leqq t_{s+1}$, and that the points $y_{h k}=y_{h}^{N}\left(u_{k}(x)\right), 0 \leqq x$ $\leqq t_{s+1}$, lie in the domain (2). Let 


$$
\mu_{N}=\max \sum_{\nu}\left|y_{\nu}^{N+1}(x)-y_{\nu}^{N}(x)\right| \quad\left(t_{s} \leqq x \leqq t_{s+1}\right) .
$$

Then, as in the preceding proof of the inequality (15), we find

$$
\mu_{N+1} \leqq\left(t_{s+1}-t_{s}\right) L m \mu_{N} \quad(N=0,1,2, \cdots) .
$$

Therefore, the infinite series $\sum \mu_{N}$ is majorized by a geometric series with ratio

$$
\left(t_{s+1}-t_{8}\right) L m<1 .
$$

It follows that there exist uniform limits

$$
y_{\nu}(x)=\lim _{N \rightarrow \infty} y_{\nu}^{N}(x) \quad(\nu=1, \cdots, n),
$$

and that this set of continuous functions $\left\{y_{\nu}(x)\right\}$ is the required set of solutions for $x_{0} \leqq x \leqq t_{s+1}$. Letting $s=0,1, \cdots, r-1$ in both this existence proof and the preceding uniqueness proof, we see that the proof of the theorem is complete.

3. The existence theorem. Before proceeding to Theorem 2, we note that Theorem 1 has the following essential feature: The number $x_{1}>0$ is restricted only by the assumption that $x_{1} \leqq p / M$, and is, therefore, independent of the magnitude of the Lipschitz constant $L$.

THEOREM 2. If we omit from the hypotheses of the preliminary theorem the restriction that the continuous functions $f_{\nu}$ satisfy a Lipschitz condition (4), then the system (1) must still possess at least one set of solutions $y_{1}(x), \cdots, y_{n}(x)$ which are defined and continuous for $x_{0} \leqq x$ $\leqq x_{1}$ and which satisfy the initial conditions (5).

Proof. The functions $f_{v}\left(x,\left\{y_{h k}\right\}\right)$ have been defined in the domain $D$ given by the inequalities (2). Let $T$ be a positive integer. We divide each one of the $m n+1$ intervals (2) into $T$ subintervals of equal length. The end points of these subintervals form in the $m n+1 \mathrm{di}-$ mensional domain $D$ a lattice consisting of $(T+1)^{m n+1}$ distinct points. Now we define on $D$ the unique "polygonal" function $f_{\nu}\left(x,\left\{y_{h k}\right\} ; T\right)$ of the variables $x, y_{11}, \cdots, y_{n m}$ which equals $f_{\nu}\left(x,\left\{y_{h k}\right\}\right)$ at the lattice points and which is a linear function of each one of the variables $x, y_{11}, \cdots$ in every $m n+1$ dimensional "rectangle"

$$
x^{\prime} \leqq x \leqq x^{\prime \prime}, y_{11}^{\prime} \leqq y_{11} \leqq y_{11}^{\prime \prime}, \cdots, y_{n m}^{\prime} \leqq y_{n m} \leqq y_{n m}^{\prime \prime}
$$

in which $\left[x^{\prime}, x^{\prime \prime}\right],\left[y_{11}^{\prime}, y_{11}^{\prime \prime}\right], \cdots$ are subintervals formed in the given subdivisions of the intervals $\left[0, x_{1}\right],\left[\eta_{11}, \zeta_{11}\right], \ldots$. In each such "rectangle" (16) the function $f_{v}\left(x,\left\{y_{h k}\right\} ; T\right)$ has an explicit repre- 
sentation as a sum of $2^{m n+1}$ terms:

$$
\sum_{\sigma,\{\tau h k} f_{\nu}\left(\sigma,\left\{\tau_{h k}\right\}\right)\left(1-\frac{|x-\sigma|}{x^{\prime \prime}-x^{\prime}}\right) \prod_{h, k}\left(1-\frac{\left|y_{h k}-\tau_{h k}\right|}{y_{h k}^{\prime \prime}-y_{h k}^{\prime}}\right),
$$

where the sum is extended over the values $\sigma=x^{\prime}, x^{\prime \prime} ; \tau_{h k}=y_{h \mathbf{k}}^{\prime}, y_{h \mathbf{k}}^{\prime \prime}$. Now the function $f_{\nu}\left(x,\left\{y_{h k}\right\} ; T\right)$ is uniquely defined as a continuous, piecewise multilinear function on $D$. Furthermore, we have

$$
\max \left|f_{\nu}\left(x,\left\{y_{h k}\right\} ; T\right)\right| \leqq \max \left|f_{\nu}\left(x,\left\{y_{h k}\right\}\right)\right| \leqq M .
$$

And, since $f_{v}\left(x,\left\{y_{h k}\right\}\right)$ is a continuous function, we have

$$
\lim _{T \rightarrow \infty} f_{\nu}\left(x,\left\{y_{h k}\right\} ; T\right)=f_{\nu}\left(x,\left\{y_{h k}\right\}\right)
$$

uniformly in the closed domain $D$. Finally, each function $f_{v}\left(x,\left\{y_{h k}\right\} ; T\right)$ satisfies a Lipschitz condition of the type (4). It follows from Theorem 1 that there must exist continuous functions $y_{1}(x ; T), \cdots$, $y_{n}(x ; T)$ satisfying the equations

$$
\begin{array}{cc}
y_{\nu}(x ; T)=y_{\nu}(0 ; T)+\int_{0}^{x} f_{\nu}\left(t,\left\{y_{h}\left(u_{k}(t) ; T\right)\right\} ; T\right) d t & \left(0 \leqq x \leqq x_{1}\right), \\
y_{\nu}(x ; T)=g_{\nu}(x) & \left(x_{0} \leqq x \leqq 0\right),
\end{array}
$$

and that these functions must satisfy the inequalities

$$
\left|y_{\nu}(x ; T)-g_{\nu}(0)\right| \leqq p \quad\left(0 \leqq x \leqq x_{1}\right) .
$$

The last inequalities and the initial conditions (20) show that the functions $y_{\nu}(x ; T)$ are uniformly bounded. The functions $y_{\nu}(x ; T)$ are also equicontinuous, for by (17), (19), and (20) we have

$$
\begin{aligned}
\mid y_{\nu}(x ; T)- & y_{\nu}\left(x^{*} ; T\right) \mid \\
& \leqq\left\{\begin{array}{lr}
M\left|x-x^{*}\right| & \left(0 \leqq x \leqq x^{*} \leqq x_{1}\right), \\
\left|g_{\nu}(x)-g_{\nu}(0)\right|+M x^{*} & \left(x_{0} \leqq x \leqq 0 \leqq x^{*} \leqq x_{1}\right), \\
\left|g_{\nu}(x)-g_{\nu}\left(x^{*}\right)\right| & \left(x_{0} \leqq x \leqq x^{*} \leqq 0\right) .
\end{array}\right.
\end{aligned}
$$

Therefore, by Ascoli's selection theorem, there exists a subsequence of integers $T_{1}, T_{2}, T_{3}, \cdots$ for which we have $n$ uniform limits

$$
\lim _{N \rightarrow \infty} y_{\nu}\left(x ; T_{N}\right)=y_{\nu}(x) \quad(\nu=1, \cdots, n) .
$$

These limit functions are continuous functions of $x$. Taking limits in (21), (20), (19) we have, by (18), 


$$
\begin{array}{rr}
\left|y_{\nu}(x)-g_{\nu}(0)\right| \leqq p & \left(0 \leqq x \leqq x_{1}\right), \\
y_{\nu}(x)=g_{\nu}(x) & \left(x_{0} \leqq x \leqq 0\right), \\
y_{\nu}(x)=y_{\nu}(0)+\int_{0}^{x} f_{\nu}\left(t,\left\{y_{h}\left(u_{k}(t)\right)\right\}\right) d t & \left(0 \leqq x \leqq x_{1}\right) .
\end{array}
$$

Since the integrand is continuous, each function $y_{v}(x)$ is continuously differentiable for $0 \leqq x \leqq x_{1}$, and the proof of the theorem is complete.

\section{REFERENCES}

1. G. Peano, Démonstration de l'intégrabilité des équations différentielles ordinaires, Math. Ann. vol. 37 (1890) pp. 182-228.

2. E. Picard, Mémoire sur la théorie des équations aux dérivées partielles et la méthode des approximations successives, J. de Math. (4) vol. 6 (1890) pp. 145-210.

3. E. Lindelöf, Sur l'application des méthodes d'approximations successives à l'étude des intégrales réelles des équations différentielles ordinaires, J. de Math. (4) vol. 10 (1894) pp. 117-128.

4. J. Franklin, On the method of successive approximations, Technical Report No. 7, Applied Mathematics and Statistics Laboratory, Stanford University, 1952.

5. E. Kamke, Differentialgleichungen reeller Funktionen, New York, 1947.

STANFORD UNIVERSITY 